\title{
Adubação de cobertura com nitrogênio, potássio e cálcio na produção comercial de cenoura
}

\author{
José Magno Q Luz; Adílio Zorzal Filho²; Welisson L Rodrigues'; Carlos R Rodrigues³; Angelica A \\ Queiroz $^{1}$ \\ ${ }^{1}$ UFU-ICA, Campus Umuarama, Rua Amazonas s/n, 38400-902 Uberlândia-MG; ${ }^{2}$ Engenheiro Agrônomo, COOPADAP, C. postal 37, \\ 38800-000 São Gotardo-MG; UFRPE-Unid. Acad. de Garanhuns, Av. Bom Pastor, s/n, 55296-901 Garanhuns-PE; jmagno@umuarama. \\ ufu.br; adilio@coopadap.com.br; carlos_rrodrigues@yahoo.com.br
}

\section{RESUMO}

A cenoura é a principal hortaliça em valor econômico cujo produto é a raiz, encontrando-se entre as dez espécies de olerícolas mais cultivadas no Brasil. O presente trabalho avaliou a produtividade, número e qualidade de raízes comerciais de cenoura, cultivar Brasília-RL, em diferentes adubações de cobertura. O experimento foi conduzido num Latossolo Vermelho-Amarelo, da Fazenda Olhos D’Água, em São Gotardo-MG, de janeiro a abril de 2006. O delineamento experimental foi em blocos casualizados com nove tratamentos $\left(\mathrm{T} 0=\right.$ testemunha sem cobertura; $\mathrm{T} 1=100 \mathrm{~kg} \mathrm{ha}^{-1}$ de 20-00-20 aos 30 e 45 dias após semeadura; T2 $=200 \mathrm{~kg} \mathrm{ha}^{-1}$ de 20-00-20 aos 30 e 45 dias após semeadura; T3 $=300 \mathrm{~kg} \mathrm{ha}^{-1}$ de 20-00-20 aos 30 e 45 dias após semeadura; T4 $=200 \mathrm{~kg} \mathrm{ha}^{-1}$ de 20-00-20 aos 30 dias após semeadura; T5=200 $\mathrm{kg} \mathrm{ha}^{-1}$ de 20-00-20 aos 45 dias após a semeadura; $\mathrm{T} 6=300 \mathrm{~kg} \mathrm{ha}^{-1}$ de 20-00-20 aos 45 dias após a semeadura; T7= $130 \mathrm{~kg} \mathrm{ha}^{-1}$ de nitrocálcio aos 30 e 45 dias após semeadura e T8=130 $\mathrm{kg} \mathrm{ha}^{-1}$ de nitrocálcio aos 30 dias após semeadura e T9=133 $\mathrm{kg} \mathrm{ha}^{-1}$ de 15-00-14 aos 45 dias após semeadura) e cinco repetições. Foram avaliados o número e a massa de raízes nas classes comerciais: $\mathrm{C}-10$ $(>10<14 \mathrm{~cm}), \mathrm{C}-14(>14<18 \mathrm{~cm}), \mathrm{C}-18(>18<22 \mathrm{~cm}), \mathrm{C}-22(>22<26$ $\mathrm{cm})$ e $\mathrm{C}-26(>26 \mathrm{~cm})$, o número e massa de raízes comerciais $(\mathrm{C})$ e não comerciais $(\mathrm{NC})$ e número total de raízes $(\mathrm{T})$. O número total de raízes de cenoura ( $\mathrm{T}$ ) não foi afetado significativamente pelos tratamentos de adubação de cobertura, sendo a máxima produtividade de raízes $\left(27,5 \mathrm{t} \mathrm{ha}^{-1}\right)$, utilizando-se o formulado 20-00-20, obtida com a aplicação de $229,1 \mathrm{~kg} \mathrm{ha}^{-1}$. A aplicação conjunta de fontes de nitrogênio, potássio e cálcio aumentou a produtividade de raízes comerciais, principalmente na classe de maior valor (C-18).

Palavras-chave: Daucus carota L., fertilizantes, nutrição mineral, produtividade e qualidade.

\section{ABSTRACT}

Top-dressing fertilization with nitrogen, potassium and calcium in the root yield of carrots

The carrot is included among the ten most economically important vegetables in Brazil. We evaluated the root yield, number and quality of commercial carrots, cultivar Brasília-RL, under different top-dressing fertilizations. The experiment was carried out on a Latosol, at Fazenda Olhos D'Água, in São Gotardo, Minas Gerais State, from January to April 2006. The experiment was set up in randomized blocks with nine treatments $(\mathrm{T} 0=$ no top-dressing control; $\mathrm{T} 1=100 \mathrm{~kg} \mathrm{ha}^{-1} 20-00-20$ at 30 and 45 days after sowing; $\mathrm{T} 2=200$ $\mathrm{kg} \mathrm{ha}^{-1} 20-00-20$ at 30 and 45 days after sowing; T3=300 $\mathrm{kg} \mathrm{ha}^{-1}$ 20-00-20 at 30 and 45 days after sowing; T4=200 $\mathrm{kg} \mathrm{ha}^{-1} 20-00-20$ at 30 days after sowing; T5 $=200 \mathrm{~kg} \mathrm{ha}^{-1} 20-00-20$ at 45 days after sowing; $\mathrm{T} 6=300 \mathrm{~kg} \mathrm{ha}^{-1} 20-00-20$ at 45 days after sowing; $\mathrm{T} 7=130$ $\mathrm{kg} \mathrm{ha}{ }^{-1}$ nitrocalcium at 30 and 45 days after sowing; $\mathrm{T} 8=130 \mathrm{~kg} \mathrm{ha}^{-1}$ nitrocalcium at 30 days after sowing and $\mathrm{T} 9=133 \mathrm{~kg} \mathrm{ha}^{-1} 15-00-14$ at 45 days after sowing) and five replications. The root number and weight in each commercial class were evaluated: $\mathrm{C}-10(>10<14 \mathrm{~cm})$, C-14 ( $>14<18 \mathrm{~cm}), \mathrm{C}-18(>18<22 \mathrm{~cm}), \mathrm{C}-22(>22<26 \mathrm{~cm}), \mathrm{C}-26(>26$ $\mathrm{cm})$, the commercial (C), the non commercial (NC) and total (T) root number and weight were also evaluated. The total number of carrot roots $(\mathrm{T})$ did not present significant differences with the top-dressing fertilizations. Maximum root yield (27.5 $\left.\mathrm{t} \mathrm{ha}^{-1}\right)$ using 20-00-20 was obtained with the fertilization with $229.1 \mathrm{~kg} \mathrm{ha}^{-1}$. However the joint application of nitrogen, potassium and calcium sources increased commercial root yield, especially of those classified as C-18, which is the major commercial class.

Keywords: Daucus carota L., fertilizer, mineral nutrition, yield and, quality.

\section{(Recebido para publicação em 8 de janeiro de 2009; aceito em 4 de dezembro de 2009)} (Received in January 8, 2009; accepted in December 4, 2009)

\begin{abstract}
$\mathrm{A}$ cenoura (Daucus carota L.), pertencente à família Apiaceae, é a principal hortaliça de raiz em valor econômico e encontra-se entre as dez espécies de olerícolas mais cultivadas no Brasil, com consumo per capita de 5,8 kg/pessoa/ano (Maranhão, 2003).

É cultivada em larga escala nas regiões Sudeste, Nordeste e Sul do Brasil, com área plantada estimada, em 2005, de 28 mil hectares e produção de 800 mil toneladas de raízes (Embrapa, 2006). Apresenta alto conteúdo de vitamina A,
\end{abstract}

textura macia e paladar agradável. Além do consumo in natura é utilizada como matéria prima para indústrias processadoras de alimentos, que a comercializam na forma de minimamente processada (minicenouras, cubos, ralada, em rodelas) ou processada na forma de salada de legumes, alimentos infantis e sopas instantâneas (Filgueira, 2003).

Dentre as cultivares de cenoura produzidas no país para cultivo de primavera-verão, a Brasília tem se destacado, sendo plantada em praticamente todo o território nacional (Nascimento \& Vieira, 1992). Segundo Filgueira (2003), as modernas cultivares são exigentes em nutrientes, que devem ser fornecidos em forma altamente assimilável.

Pesquisas conduzidas no Brasil mostram que a extração de macronutrientes pela cenoura apresenta a seguinte ordem decrescente: K, N, Ca, P, S e Mg. De maneira geral a adubação de $\mathrm{N}$ e K é feita em quase sua maioria em cobertura e o fornecimento de Ca é creditado somente à aplicação de calcário e gesso (Raij et 
al., 1997; Ribeiro et al., 1999). Experimentalmente, tem-se obtido maiores produtividade da cenoura aplicando o $\mathrm{K}$ na adubação de cobertura (Filgueira, 2003). O mesmo autor relata que são poucas as olerícolas que respondem à aplicação do K em adubação de cobertura, sendo a cenoura uma delas. Nem sempre a cultura da cenoura tem respondido à adição de cálcio, mas o principal efeito desse nutriente sobre a produção da cenoura é indireto através da relação Ca: $\mathrm{Mg}$ do solo.

No estado de Minas Gerais, para uma produção esperada de 35 a $40 \mathrm{t}$ ha $^{-1}$ recomenda-se aplicação de NPK levando em consideração a textura do solo e a disponibilidade de fósforo ou de potássio. Sugere-se a aplicação de $100 \%$ do fósforo, $30 \%$ do nitrogênio e $40 \%$ do potássio no plantio, e o restante do nitrogênio e potássio (70 e $60 \%$ respectivamente) deve ser aplicado em duas coberturas, aos 20 e aos 40 dias da emergência, adicionando ainda no plantio 1 a $2 \mathrm{~kg} \mathrm{ha}^{-1}$ de boro e, ou, 2 a $3 \mathrm{~kg}$ $\mathrm{ha}^{-1}$ de zinco, caso o solo seja deficiente (Ribeiro et al., 1999).

A adubação em cobertura normalmente deve ser feita com $40 \mathrm{~kg} \mathrm{ha}^{-1}$ de nitrogênio (N). Entretanto, nos plantios em épocas chuvosas, recomenda-se a aplicação de $60 \mathrm{~kg} \mathrm{ha}^{-1}$ de $\mathrm{N}$ e $60 \mathrm{~kg} \mathrm{ha}^{-1}$ de $\mathrm{K}_{2} \mathrm{O}$, aos 30 e 60 dias após a emergência. Normalmente, quando se incorpora o esterco de galinha na dosagem recomendada, a adubação de cobertura com nitrogênio pode ser dispensada, se o desenvolvimento das plantas for normal (Vieira \& Makishima, 2000).

Oliveira et al. (2001), comparando a produção de raízes de cenoura cultivadas com húmus de minhoca e adubo mineral, aplicaram no plantio $800 \mathrm{~kg} \mathrm{ha}^{-1}$ de superfosfato simples e $136 \mathrm{~kg} \mathrm{ha}^{-1} \mathrm{de}$ cloreto de potássio e, em cobertura 200 $\mathrm{kg} \mathrm{ha}^{-1}$ de sulfato de amônia, em duas parcelas iguais aplicadas aos 30 e 60 dias após semeadura, obtendo produção comercial de $25,5 \mathrm{t} \mathrm{ha}^{-1}$ de raízes. Isso representa $64,7 \%$ de superioridade na produção comercial quando comparada com a ausência da adubação mineral. Este resultado expressa a exigência da cultura em adubo mineral para obtenção de elevados rendimentos.

Tendo em vista não haver na literatu- ra nacional informações sobre fontes de $\mathrm{N}$ e K em cobertura, o presente trabalho teve como principal objetivo avaliar a produtividade e a qualidade comercial de raízes de cenoura, quando submetidas a diferentes adubações de cobertura.

\section{MATERIAL E MÉTODOS}

O experimento foi conduzido entre 17/01 e 27/04/2006 numa área comercial no município de São Gotardo-MG. Os dados georeferenciais apresentam os seguintes valores: altitude $=1.100$ $\mathrm{m}$; latitude $=19^{\circ} 12^{\prime} 58^{\prime}$ ' $\mathrm{S}$ e longitude $=$ 46 ${ }^{\circ} 14^{\prime} 02$ " WO. O clima, segundo a classificação de Köppen é Aw, clima tropical, temperatura média anual de $18^{\circ} \mathrm{C}$ e precipitação em torno de 850 $\mathrm{mm}$, sendo a unidade predominante de solo o Latossolo vermelho-amarelo distrófico (Embrapa, 1999). As características químicas do solo na camada de $0-15$ cm foram: $\mathrm{pH} \mathrm{H}_{2} \mathrm{O}=5,2 ; \mathrm{P}=$ $57 \mathrm{mg} \mathrm{dm}^{-3} ; \mathrm{K}=5,9 \mathrm{mmol}_{\mathrm{c}} \mathrm{dm}^{-3} ; \mathrm{Ca}=$ $46 \mathrm{mmol}_{\mathrm{c}} \mathrm{dm}^{-3} ; \mathrm{Mg}=13 \mathrm{mmol} \mathrm{dm}^{-3}$; $\mathrm{Na}=0,2 \mathrm{mmol}_{\mathrm{c}} \mathrm{dm}^{-3} ; \mathrm{Al}=1 \mathrm{mmol}_{\mathrm{c}} \mathrm{dm}^{-3}$ e $\mathrm{V}=63 \%$.

Foi utilizada a cultivar Brasília-RL; a semeadura foi realizada mecanicamente em 18/01, na profundidade de 1,5 a 2,0 cm. A adubação de plantio foi realizada no dia anterior, sendo aplicado $2.500 \mathrm{~kg} \mathrm{ha}^{-1}$ da formulação 04-30-16 +0,2\% de $\mathrm{Zn}+0,3 \%$ de B e $800 \mathrm{~kg} \mathrm{ha}^{-1}$ de superfosfato simples. $\mathrm{Na}$ adubação de cobertura foram utilizados os formulados 20-00-20 (20\% de $\mathrm{N}$ e $20 \%$ de $\mathrm{K}_{2} \mathrm{O}$ ), $15-00-14$ (15\% N e $14 \%$ de $\left.\mathrm{K}_{2} \mathrm{O}\right)$ e nitrocálcio $(15,5 \%$ de $\mathrm{N}$ e $17 \%$ de $\mathrm{Ca}$ ).

A área total da parcela foi de 4,80 $\mathrm{m}^{2}(3,0 \times 1,6 \mathrm{~m})$ contendo seis linhas duplas longitudinais, com espaçamento nas entrelinhas de $15 \mathrm{~cm}$ e dentro das linhas de $10 \mathrm{~cm}$ entre plantas, após o desbaste, que foi realizado aos 30 dias após a semeadura.

O delineamento do experimento foi em blocos casualizados, com cinco repetições, sendo os tratamentos constituídos de quatro doses do fertilizante formulado NPK 20-00-20 (0, 100, 200 e 300 kg $\left.\mathrm{ha}^{-1}\right)$, parceladas com duas aplicações, aos 30 e 45 dias após semeadura (DAS) e cinco tratamentos adicionais, sendo
$300 \mathrm{~kg} \mathrm{ha}^{-1}$ do formulado 20-00-20 aos 45 DAS; $200 \mathrm{~kg} \mathrm{ha}^{-1}$ do formulado 20 00-20 aos 30 DAS; $200 \mathrm{~kg} \mathrm{ha}^{-1}$ do formulado NPK 20-00-20 aos 45 DAS; 130 $\mathrm{kg} \mathrm{ha}^{-1}$ de nitrocálcio parcelados aos 30 e 45 DAS, e $130 \mathrm{~kg} \mathrm{ha}^{-1}$ de nitrocálcio + $133 \mathrm{~kg} \mathrm{ha}^{-1}$ do formulado NPK 15-00-14 parcelados aos 30 e 45 DAS, respectivamente. Foram realizadas adubações foliares a partir do $30^{\circ}$ dia após o plantio, com produtos contendo os micronutrientes $\mathrm{Mn}, \mathrm{Zn}$ e B e, também o $\mathrm{Ca}$, tendo como fontes e doses respectivamente, $\mathrm{MnCl}_{2}$ (400 mL/há, uma aplicação aos 40 dias após a semeadura (DAS). e outra aos 60 DAS.), $\mathrm{ZnSO}_{4}$ (300 mL/há, uma aplicação aos 45 DAS) e $\mathrm{CaB}^{\circledR}$ (1.500 $\mathrm{mL} /$ há, parcelado em quatro aplicações: a primeira aos 40 DAS seguida de três outras a cada sete dias).

O manejo de plantas daninhas foi feito em pré e pós emergências (30 dias após o plantio), através do uso de herbicidas à base de afalon, select e lanzar, sendo o controle de doenças realizado semanalmente, com o uso de fungicidas à base de azoxytrobin, captam, difenoconazole, mancozeb, oxicloreto de cobre e hidróxido de cobre. Em relação ao manejo de irrigação não foi utilizado nenhum parâmetro técnico, apenas a experiência do produtor e precipitações naturais.

A colheita foi realizada manualmente em 2,50 $\mathrm{m}^{2}$ da área útil de cada parcela (quatro linhas duplas centrais) aos 100 DAS. As raízes colhidas foram lavadas e classificadas em cinco classes comerciais: C-10 ( $>10 \mathrm{~cm}<14 \mathrm{~cm})$, C-14 $(>14 \mathrm{~cm}<18 \mathrm{~cm})$, C-18 $(>18 \mathrm{~cm}<22$ $\mathrm{cm}), \mathrm{C}-22(>22 \mathrm{~cm}<26 \mathrm{~cm})$ e C-26 $(>26$ $\mathrm{cm})$ e uma não comercial $(\leq 10 \mathrm{~cm}$ e/ou com defeito), conforme proposto pelo Programa Brasileiro Para a Melhoria dos Padrões Comerciais e Embalagens implantado pela Companhia de Entrepostos e Armazéns Gerais de São Paulo (CEAGESP, 1999), sendo posteriormente contadas e pesadas.

Os dados referentes às variáveis, número de raízes e produtividade $\left(\mathrm{kg} \mathrm{ha}^{-1}\right)$ foram submetidos à análise de variância com teste de $\mathrm{F}$ ao nível de 1 e $5 \%$ de significância, com auxílio do programa SISVAR (Ferreira, 2000).

Foi realizada análise de regressão em função das doses crescentes do for- 
mulado 20-00-20, teste de média (Scott Knott a 5\%) entre os tratamentos adicionais e para comparação entre as doses crescentes do formulado 20-00-20 e os tratamentos adicionais foram realizados contrastes, que foram comparados pelo teste de F, com auxílio do programa SISVAR (Ferreira, 2000).

\section{RESULTADOS E DISCUSSÃO}

Não houve efeito da adubação de cobertura no número total de raízes de cenoura $(\mathrm{T})$. No entanto, para o número total de raízes comercializáveis (C) houve efeito da aplicação de cobertura de doses crescentes do fertilizante formulado 20-00-20 ( $\left.-\mathrm{P}_{2} \mathrm{O}_{5}-\mathrm{K}_{2} \mathrm{O}\right)$ e dos tratamentos adicionais.

$\mathrm{O}$ número de raízes comerciais de cenoura apresentou ajuste linear (Figura 1) em função das doses crescentes do fertilizante 20-00-20. Como a maior dose do fertilizante não proporcionou a máxima produtividade (Figura 1) e com o objetivo de comparação com os tratamentos adicionais, adotou-se o número de raízes produzidas no tratamento com a aplicação de $300 \mathrm{~kg} \mathrm{ha}^{-1}$ do formulado 20-00-20 (121,2 raízes parcela-1 $)$ para comparar com os resultados dos tratamentos adicionais. Entre os tratamentos adicionais foi observado que a aplicação de $130 \mathrm{~kg} \mathrm{ha}^{-1}$ de nitrocálcio aos 30 dias de cultivo e $133 \mathrm{~kg} \mathrm{ha}^{-1}$ do formulado 15-00-14, aos 45 dias de cultivo, proporcionaram maior número de raízes comerciais por parcela (Tabela 1).

A Figura 2 apresenta a correlação entre o número de raízes comerciais e as doses de N (Figura 2A) e de K (Figura 2B) aplicados, também com uso de $\mathrm{Ca}$, onde observa-se que não houve significância das correlações. Retirando-se os tratamentos à base de $\mathrm{Ca}$, observou-se que há correlação positiva e significativa entre o número de raízes e as doses crescentes de $\mathrm{N}$ e $\mathrm{K}$ (Figuras 2C e 2D, respectivamente). Esses resultados indicam que o diferencial no presente experimento foi a adição de $\mathrm{Ca}$ em cobertura.

Dentre as classes comerciais, somente para o número de raízes classificadas como C-18, houve variação significativa entre os tratamentos. Também não
Tabela 1. Estimativa de contraste para o número de raízes comercial (C) e da classe C-18 e produção de raízes $\left(\mathrm{kg} \mathrm{ha}^{-1}\right)$ geral e da classe C-18 (estimated contrast of the number of roots of commercial (C) and class $(\mathrm{C}-18)$, and roots production $\left(\mathrm{kg} \mathrm{ha}^{-1}\right)$ in the whole and in the class C-18). Uberlândia, UFU, 2006.

\begin{tabular}{|c|c|c|c|c|}
\hline \multirow{2}{*}{ Contraste } & \multicolumn{2}{|c|}{ Produtividade (t ha $\left.{ }^{-1}\right)$} & \multicolumn{2}{|c|}{ Raízes } \\
\hline & C-18 & Total & C-18 & Total \\
\hline Regressão vs. adicionais & $-17,4^{*}$ & $-705,36^{\mathrm{ns}}$ & $-6,25^{*}$ & $-8,38^{*}$ \\
\hline $\begin{array}{l}300 \mathrm{~kg} \mathrm{ha}^{-1}(20-00-20) \mathrm{vs} . \\
\text { nitrocálcio e } 15-00-14\end{array}$ & $-2.832,80 * *$ & $-2.056,80^{\mathrm{ns}}$ & $-14,6^{* *}$ & $-17,4^{*}$ \\
\hline
\end{tabular}

houve variação significativa para o total não comercializável (NC). A adubação de cobertura pode não ter influenciado no número total de raízes, mas alterou significativamente a quantidade de raízes de melhor qualidade. Na Tabela 1, observa-se, que a resposta se deve aos tratamentos. Houver aumento significativo com a aplicação de nitrocálcio e formulado 15-00-14 para o número total de raízes comerciais e da classe C-18.

Dentro de cada grupo de tratamentos, selecionando o que proporcionou os maiores valores, $300 \mathrm{~kg} \mathrm{ha}^{-1}$ do formulado 20-00-20 vs. nitrocálcio + formulado 15-00-14, observou-se que a aplicação do último proporcionou valores superiores tanto para o número total de raízes comerciais $(\mathrm{C})$ quanto para a classe C-18 (Tabela 2). O nitrocálcio isoladamente não foi o responsável pelo acréscimo, mais sim a sua mistura com o formulado 15-00-14.
Em geral, os resultados apresentados para o número total de raízes comerciais são semelhantes aos observados para o número de raízes da classe $\mathrm{C}-18$. Na Figura 2 observa-se um comportamento semelhante nas correlações entre as doses de $\mathrm{N}$ e $\mathrm{K}$ e o número total e da classe C-18 de raízes comerciais, evidenciando a importância da inclusão do Ca na nutrição da cenoura.

A produtividade de raízes $\left(\mathrm{kg} \mathrm{ha}^{-1}\right)$ apresentou, de maneira geral, resultados semelhantes aos observados para o número de raízes, com exceção da produtividade total $(\mathrm{T})$ de raízes $(\mathrm{kg}$ $\mathrm{ha}^{-1}$ ) onde não houve diferença entre os tratamentos principais e os adicionais (Tabela 1). A produtividade de raízes comercializáveis (C) foi alterada significativamente somente com as doses crescentes do fertilizante 20-00-20 (Tabela 1). Dentre as classes comerciais, o resultado foi semelhante ao observado
Tabela 2. Produtividade $\left(\mathrm{kg} \mathrm{ha}^{-1}\right)$ e número de raízes de cenoura da classe C-18, cv. BrasíliaRL, em função da aplicação de fertilizantes em cobertura (yield and number of carrot roots of the class C-18, cv. Brasilia-RL, depending on the application of fertilizers in coverage). Uberlândia, UFU, 2006.

\begin{tabular}{|c|c|c|c|c|c|}
\hline \multirow{2}{*}{ Fertilizantes } & \multirow{2}{*}{$\begin{array}{c}\text { Doses } \\
\left(\mathrm{kg} \mathrm{ha}^{-1}\right)\end{array}$} & \multirow{2}{*}{$\begin{array}{c}\text { Época(s) } \\
(\text { dias })^{1}\end{array}$} & \multicolumn{2}{|c|}{ Raízes (número) } & \multirow{2}{*}{$\frac{\text { Raízes }\left(\mathrm{kg} \mathrm{ha}^{-1}\right)}{\mathrm{C}-18}$} \\
\hline & & & Total C & C-18 & \\
\hline nitrocálcio & 130 & 30 e 45 & $118,60 b^{4}$ & $44,00 \mathrm{~b}$ & $8.832,0 \mathrm{~b}^{3}$ \\
\hline $\begin{array}{l}\text { nitrocálcio }{ }^{1} \text { e } \\
15-00-14^{2}\end{array}$ & $130^{2}$ e $133^{3}$ & 30 e 45 & $138,60 \mathrm{a}$ & $58,80 \mathrm{a}$ & $12.080,8 \mathrm{a}$ \\
\hline $20-00-20$ & 200 & 30 & $107,00 \mathrm{~b}$ & $36,40 \mathrm{~b}$ & $8.220,0 \mathrm{~b}$ \\
\hline $20-00-20$ & 200 & 45 & $119,20 \mathrm{~b}$ & $48,00 \mathrm{~b}$ & $10.116,0 \mathrm{~b}$ \\
\hline $20-00-20$ & 300 & 45 & $111,00 \mathrm{~b}$ & $40,80 \mathrm{~b}$ & $8.844,0 \mathrm{~b}$ \\
\hline
\end{tabular}

${ }^{1}$ Época(s) de Aplicação (dias após semeadura); ${ }^{2}$ aplicado 30 dias após semeadura; ${ }^{3}$ aplicado 45 dias após a semeadura; ${ }^{4}$ médias seguidas de mesma letra na coluna não diferem entre si (Scott Knott 5\%) ( ${ }^{1}$ applied 30 days after sowing; ${ }^{2}$ applied 45 days after sowing, ${ }^{3}$ means followed by the same letter in the column do not differ from each other (Scott Knott 5\%). 


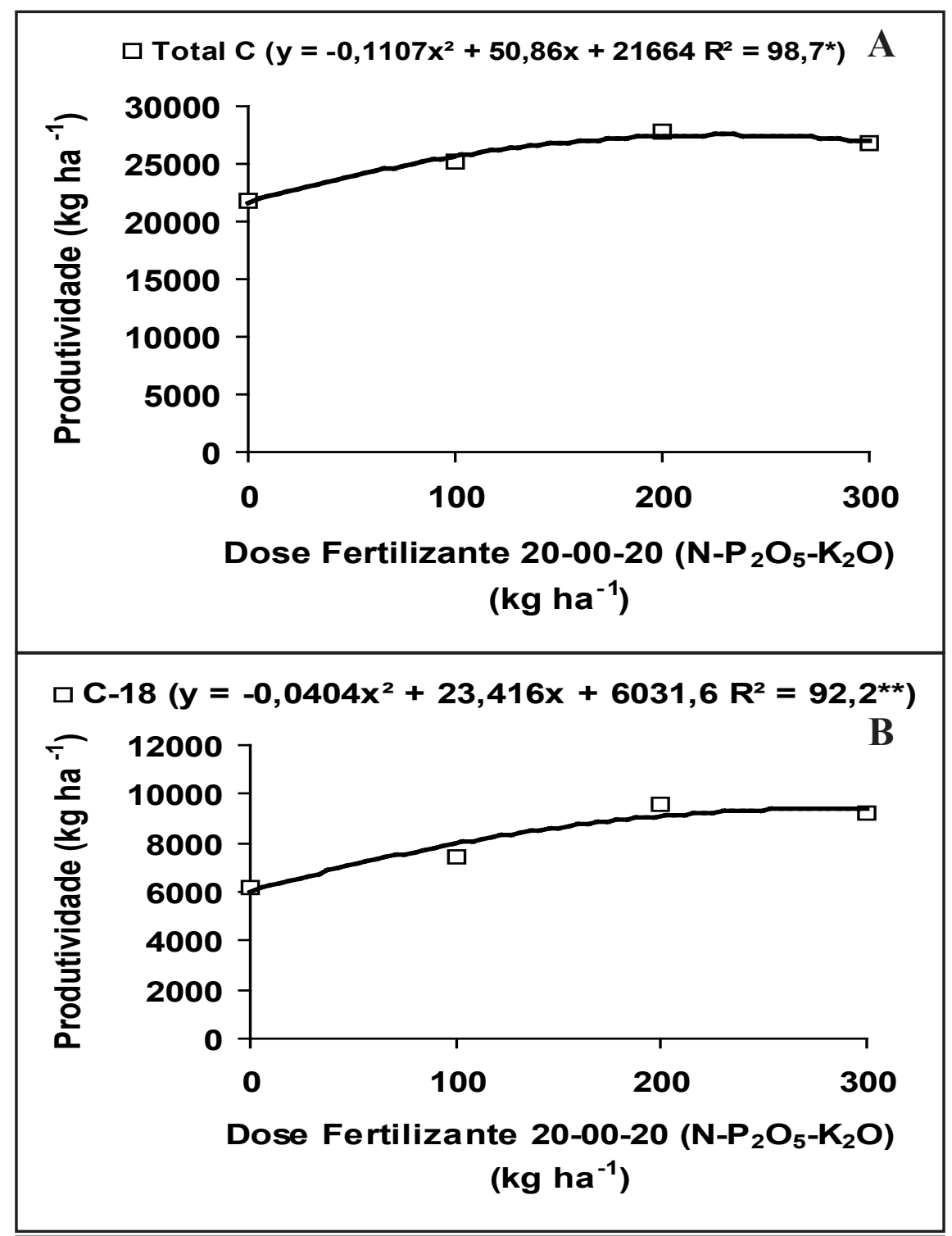

Raiz Comercial (C) $\left(y=0,0788 x+98,68 R^{2}=90,8^{\star}\right)$

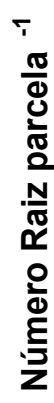

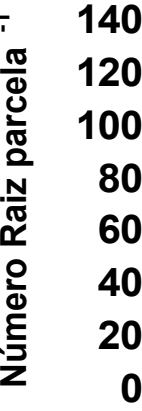
0 0 100 200 300

Dose Fertilizante 20-00-20 $\left(\mathrm{N}^{-} \mathrm{P}_{2} \mathrm{O}_{5}-\mathrm{K}_{2} \mathrm{O}\right)\left(\mathrm{kg} \mathrm{ha}^{-1}\right)$

Figura 1. Produtividade de raízes de cenoura Total comercial C(A) e total da classe C-18 (B), e número de raízes de cenoura comercial C (C) cv. Brasília-RL, em função de doses do fertilizante 20-00-20 aplicado em cobertura (yield of roots, total of commercial roots $C(A)$ and total for the class C-18 (B), and numbers of commercial carrots roots C (C), cv. Brasilia-RL, in function of fertilizer doses 20-00-20 applied in coverage) Uberlândia, UFU, 2006. para o número de raízes, sendo que a produtividade de raízes classificadas como C-18, foi alterada significativamente com todas as fontes de variação, e C-14 apresentou alteração significativa somente em função das doses crescentes do fertilizante 20-00-20.

A Tabela 1 apresenta a estimativa dos contrastes para a produtividade de raízes $\left(\mathrm{kg} \mathrm{ha}^{-1}\right)$ entre os tratamentos das doses crescentes do formulado 20-0020 e os tratamentos adicionais e com base nos melhores tratamentos de cada grupo. Para a produção total não foi verificada diferença entre os tratamentos. No entanto a classe C-18 apresentou diferenciação sendo que a aplicação de nitrocálcio + formulado 15-00-14 apresentou o maior valor, evidenciando o incremento da produtividade com a aplicação conjunta, em cobertura, do $\mathrm{Ca}, \mathrm{N}$ e K.

A produção total de raízes comerciais (C) foi alterada significativamente com as doses crescentes do formulado 20-00-20 apresentando ajuste quadrático (Figura $1 \mathrm{~A}$ ). Assim pode-se dizer que a máxima produtividade de raízes $(27,5$ $\mathrm{t} \mathrm{ha}^{-1}$ ) foi obtida com a aplicação de $229,1 \mathrm{~kg} \mathrm{ha}^{-1}$ do formulado 20-00-20. No entanto esse resultado não difere significativamente da produtividade de raízes comerciais obtidas com a aplicação dos tratamentos adicionais. Não foi observada diferença significativa de produção comercial entre todos os tratamentos do experimento e também para o contraste entre os tratamentos de dose crescente do formulado $20-00-20$ e os adicionais.

Dentre as classes comerciais observou-se que a produtividade de raízes classificadas como C-18 apresentou ajuste quadrático, sendo a máxima produtividade $\left(9,4 \mathrm{t} \mathrm{ha}^{-1}\right)$ obtida com 298,9 $\mathrm{kg} \mathrm{ha}^{-1}$ do formulado 20-00-20 (Figura 1B). Observou-se, também que a máxima produtividade de raízes da classe C-18 representou 34\% do total de raízes comerciais. Assim, pode-se inferir que o aumento da produção de raízes comerciais foi um reflexo do aumento da produtividade de raízes classificadas como C-18, ou seja, o aumento das doses do formulado 20-00-20 pode não ter alterado a produtividade total, mas interferiu na qualidade do produto 


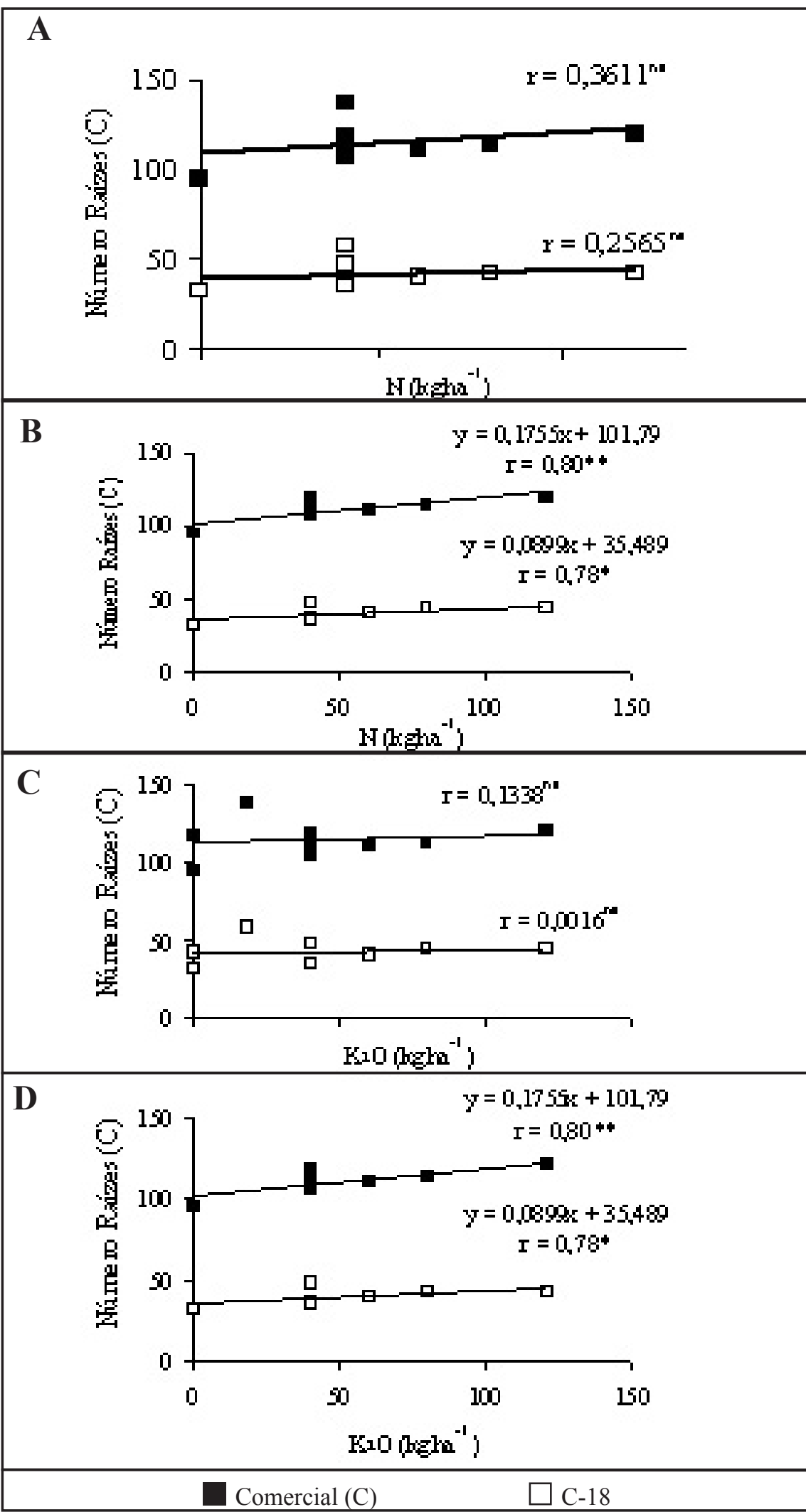

Figura 2. Correlação entre o número de raízes comerciais (C) vs. as doses de nitrogênio e potássio com os tratamentos com aplicação de $\mathrm{Ca}$ (A e B, respectivamente) e vs. as doses de nitrogênio e potássio sem os tratamentos com adição de $\mathrm{Ca}(\mathrm{C}$ e $\mathrm{D}$, respectivamente), para o número de raízes comerciais $(\mathrm{C})$ e da classe $\mathrm{C}-18$ (correlation between the number of commercial roots (C) vs. the doses of nitrogen and potassium with the treatments with application of $\mathrm{Ca}$ (A and $\mathrm{B}$, respectively) and vs. the doses of nitrogen and potassium without the treatments with the addition of $\mathrm{Ca}(\mathrm{C}$ and $\mathrm{D}$, respectively) for the number of commercial roots (C) and class C-18)). Uberlândia, UFU, 2006. produzido, aumentado a proporção do total de raízes comerciais (Figura $1 \mathrm{~A}$ ) e principalmente da classe $\mathrm{C}-18$ (Figura $1 \mathrm{~B})$.

Observou-se que para a produtividade de raízes da classe $\mathrm{C}-18$ a aplicação dos tratamentos adicionais proporcionou aumento de produção significativa somente no tratamento com a aplicação conjunta de $\mathrm{N}, \mathrm{K}$ e $\mathrm{Ca}$ em cobertura (Tabela 2). Comparando a média de produção de raízes com aplicação dos tratamentos com doses crescentes do formulado 20-00-20 e os tratamentos adicionais observa-se aumento significativo apenas com aplicação conjunta de $\mathrm{N}, \mathrm{K}$ e Ca em cobertura (Tabela 2).

A diferença básica entre os tratamentos $300 \mathrm{~kg} \mathrm{ha}^{-1}$ de 20-00-20 e nitrocálcio + 15-00-14, tanto para o número quanto para a produtividade de raízes da classe $\mathrm{C}-18$ (Tabela 1) se deve às doses de $\mathrm{Ne}$ $\mathrm{K}\left(\mathrm{kg} \mathrm{ha}^{-1}\right)$ aplicada e a presença ou ausência de $\mathrm{Ca}$, como pode ser observado na Tabela 2. Mesmo a quantidade de $\mathrm{N}$ e $\mathrm{K}$ sendo inferior no tratamento com a aplicação do nitrocálcio + formulado 1500-14 em relação a duas aplicações de $300 \mathrm{~kg} \mathrm{ha}^{-1}$ de 20-00-20, esse tratamento apresentou maior número e produtividade de raízes da classe C-18 (Tabela 1). Essa diferença pode estar relacionada com o maior suprimento de $\mathrm{Ca}$, que por sua vez está diretamente relacionado ao crescimento e à resistência do sistema radicular das plantas (Marschner, 1995; Malavolta et al., 1997; Mengel \& Kirkby, 2001; Faquin \& Andrade, 2004; Epstein \& Bloom, 2006). Mesmo o solo apresentando altos teores de $\mathrm{Ca}$ antes do plantio (46 mmolc dm ${ }^{-3}$ ), a aplicação de $130 \mathrm{~kg}$ de nitrocálcio adicionou $22,1 \mathrm{~kg}$ $\mathrm{ha}^{-1} \mathrm{de} \mathrm{Ca}$, que pode ter proporcionado toda a diferença na produtividade.

Na Tabela 2 observa-se que a aplicação somente de nitrocálcio não proporcionou efeito semelhante à aplicação de fontes que forneçam conjuntamente $\mathrm{Ca}, \mathrm{K}$ e N. Assim, pode-se afirmar que a aplicação conjunta em cobertura de fontes de $\mathrm{Ca}, \mathrm{K}$ e $\mathrm{N}$ pode proporcionar produto final de melhor qualidade. Como relatado anteriormente, o Ca é um dos principais nutrientes relacionados ao crescimento do sistema radicular, aumentando a rigidez das paredes celulares participando da formação da lamela 
média. O K é o principal nutriente relacionado à qualidade de produção e/ou do produto, pois esse nutriente participa na ativação de várias enzimas durante a biosíntese de fotoassimilados, transporte de carboidratos da fonte (folha) para os reservatórios ou drenos (raízes) e ativador enzimático da sintetase do amido (Marschner, 1995; Faquin \& Andrade, 2004; Taiz \& Zeiger, 2004; Epstein \& Bloom, 2006).

A resposta significativa da adição do $\mathrm{Ca}$ à adubação de cobertura da cenoura, cultivar Brasília-RL, pode estar relacionada a vários aspectos, como, por exemplo, a relação $\mathrm{Ca}: \mathrm{Mg}$ no solo antes do plantio. Essa relação no solo antes do plantio no presente trabalho foi igual a 3,5. Como relatado por Filgueira (2003) o Mg é um dos macronutrientes menos exigidos pela cenoura e o $\mathrm{Ca}$ o terceiro mais exigido, sendo absorvido em quantidades inferiores somente ao K e N. Lima et al. (1980) relatam que a cenoura apresentou alta produção em solos com relação $\mathrm{Ca}: \mathrm{Mg}$ próxima a $12: 1$, e isso pode ser um reflexo da alta exigência dessa planta pelo Ca. Assim, provavelmente, cultivos em solos com relação $\mathrm{Ca}: \mathrm{Mg}$ inferior a 12:1 pode ter a produção limitada pela deficiência de Ca. Portanto, serão necessários trabalhos que comprovem a relação de $\mathrm{Ca}: \mathrm{Mg}$ ideal para a cultura da cenoura.

De maneira geral, indica-se para a maioria das culturas a relação $\mathrm{Ca}: \mathrm{Mg}$ entre 3 e 4 (Raij et al., 1997). Os resultados da análise do solo utilizado neste estudo antes do cultivo mostram que a relação está na faixa indicada por essa literatura, no entanto abaixo da relação que proporcionou maior produção con- forme relato de Lima et al. (1980).

A alteração da relação $\mathrm{Ca}: \mathrm{Mg}$ pode proporcionar efeitos negativos na nutrição e crescimento das plantas. Se o equilíbrio da relação $\mathrm{Ca}: \mathrm{Mg}$ não for adequado, há condições para a deficiência induzida de um dos nutrientes, como conseqüência de antagonismos na absorção, conforme definido por Mengel \& Kirkby (2001) como competição entre íons tipo antagonismo de cátions. Assim, há necessidade de estudos básicos para definir a relação $\mathrm{Ca}: \mathrm{Mg}$ ótima para a cenoura e se realmente existe a necessidade de complementação com o fornecimento de Ca via adubação de cobertura.

A aplicação conjunta de fontes de nitrogênio, potássio e cálcio, aumentou a produtividade de raízes comerciais, principalmente de raízes classificadas como C-18.

\section{REFERÊNCIAS}

CEAGESP. 1999. Classificação de cenoura: programa de adesão voluntária, São Paulo: Programa Horti \& Fruti, Folder.

EMPRESA BRASILEIRA DE PESQUISA AGROPECUÁRIA. Centro Nacional de Pesquisa de Solo. 1999. Sistema Brasileiro de Classificação de Solos. Brasília: EMBRAPA, SPI. 412p.

EMPRESA BRASILEIRA DE PESQUISA AGROPECUÁRIA. Centro Nacional de Pesquisa de Hortaliças. Cenoura. Disponível em: <http//:www.embrapa.cnph.com.br>. Acesso em 3 jul. 2006.

EPSTEIN E; BLOOM A. 2006. Nutrição mineral de plantas: princípios e perspectivas. Londrina: Planta. 403p.

FAQUIN V; ANDRADE AT. 2004. Nutrição mineral e diagnose do estado nutricional das hortaliças. Lavras: UFLA/FAEPE. 88p.

FERREIRA DF. Sistema Sisvar para análises estatísticas. Lavras: UFLA. 2000. Disponível em: <http://www.dex.ufla.br/ danielff/dff02. htm>. Acesso em 20 dez. 2006.

FILGUEIRA FAR. 2003. Novo manual de olericultura: agrotecnologia moderna na produção e comercialização de hortaliças. 3 ed.Viçosa: UFV. 412p.

LIMA JA; FONTES RR; VIEIRA JV; SOUZA AF. 1980. Efeito da relação $\mathrm{Ca}^{2+} / \mathrm{Mg}^{2+} \mathrm{em}$ diferentes níveis de calagem em solo do cerrado para a cultura da cenoura (Daucus carota L.). In: CONGRESSO BRASILEIRO DE OLERICULTURA, 20., 1980, Brasília. Anais...Brasília. p.93.

MALAVOLTA E; VITTI GC; OLIVEIRA SA. 1997. Avaliação do estado nutricional das plantas: princípios e aplicações. 2.ed. Piracicaba: POTAFÓS.319p.

MARANHÃO (Estado). Sebrae Maranhão. Maranhão: diagnóstico hortaliças. Disponível em: <http://.sebraema.com.br/agroneg/pages/ pesquisa/page_horta_cenoura.htm $>$. Acesso em 09 mar. $200 \overline{3}$.

MARSCHNER H. 1995. Mineral nutrition of higher plants. London: Academic Press. 889 p.

MENGEL K; KIRKBY EA. 2001. Principles of plant nutrition. 5.ed. Dodrecht: Kluwer Academic Publishers. 864p.

NASCIMENTO WM; VIEIRAJV. 1992. Avaliação da qualidade de sementes de cenoura cv. Brasília comercializadas em Brasília-DF. Horticultura Brasileira 10: 40-41.

OLIVEIRA AP; ESPÍNOLA FEJ; ARAÚJO JS; COSTA CC. 2001. Produção de raízes de cenoura cultivadas com húmus de minhoca e adubo mineral. Horticultura Brasileira 19: 77-80.

RAIJ B; CANTARELLA H; QUAGGIO JA; FURLANI AMC. 1997. Recomendações de adubação e calagem para o estado de São Paulo. 2.ed. Campinas: IAC. 285p.

RIBEIRO AC; GUIMARÃES PTG; ALVAREZ VVH. 1999. Recomendações para o uso de corretivos efertilizantes em Minas Gerais, $5^{\mathrm{a}}$ Aproximação. Viçosa: UFV. 359 p.

TAIZ L; ZEIGER E. 2004. Fisiologia vegetal. Tradução de ER. SATARÉM. 3.ed. Porto Alegre: ARTMED. 719p.

VIEIRA JV; MAKISHIMA N. 2000. Cultivo da Cenoura. Brasília: CNPH. (Sistema de Produção, 2). 